\title{
Pathology of Inhalation Anthrax in Cynomolgus Monkeys (Macaca fascicularis)
}

\author{
Daphne Vasconcelos, Roy Barnewall, Michael Babin, Robert Hunt, James Estep, \\ Carl Nielsen, Robert Carnes, and John Carney
}

Battelle (DV, RB, MB, RH, JE), Columbus, Ohio; Consultant to (CN) Defense Sciences Office (CN, JC), Defense Advanced Research Projects Agency, Arlington, Virginia; Office of Product Development and Regulatory Affairs (CN), United States Army Medical Research Institute of Infectious Diseases (CN), Fort Detrick, Maryland; and Biosciences International, Inc. (RC), San Antonio, Texas

\begin{abstract}
SUMMARY: Anthrax is considered a serious biowarfare and bioterrorism threat because of its high lethality, especially by the inhalation route. Rhesus macaques (Macaca mulatta) are the most commonly used nonhuman primate model of human inhalation anthrax exposure. The nonavailability of rhesus macaques necessitated development of an alternate model for vaccine testing and immunologic studies. This report describes the median lethal dose $\left(\mathrm{LD}_{50}\right)$ and pathology of inhalation anthrax in cynomolgus macaques (Macaca fascicularis). Gross and microscopic tissue changes were reviewed in 14 cynomolgus monkeys that died or were killed after aerosol exposure of spores of Bacillus anthracis (Ames strain). The $\mathrm{LD}_{50}$ and $95 \%$ confidence intervals were $61,800(34,000$ to 110,000$)$ colony-forming units. The most common gross lesions were mild splenomegaly, lymph node enlargement, and hemorrhages in various organs, particularly involving the meninges and the lungs. Mediastinitis, manifested as hemorrhage or edema, affected $29 \%$ of the monkeys. Microscopically, lymphocytolysis occurred in the intrathoracic lymph nodes and spleens of all animals, and was particularly severe in the spleen and in germinal centers of lymph nodes. Hemorrhages were common in lungs, bronchial lymph nodes, meninges, gastrointestinal tract, and mediastinum. These results demonstrate that the Ames strain of $B$. anthracis is lethal by the inhalation route in the cynomolgus macaque. The $\operatorname{LD}_{50}$ of the Ames strain of $B$. anthracis was within the expected experimental range of previously reported values in the rhesus monkey in an aerosol challenge. The gross and microscopic pathology of inhalation anthrax in the cynomolgus monkey is remarkably similar to that reported in rhesus monkeys and humans. The results of this study are important for the establishment of an alternative nonhuman primate model for evaluation of medical countermeasures against inhalational anthrax. (Lab Invest 2003, 83:1201-1209).
\end{abstract}

$A$ nthrax is caused by Bacillus anthracis, a Grampositive, aerobic or facultative anaerobic, rodshaped spore-forming bacterium. Infection follows dermal, gastrointestinal, or inhalation routes of entry, each with slightly different clinical manifestations; however, the final outcome, regardless of portal of entry, is often septicemia. The inhalation route is most lethal, with nonspecific initial clinical signs such as fever, malaise, headache, nausea, and vomiting. The spores are phagocytosed by pulmonary macrophages and germinate in the draining mediastinal and thoracic lymph nodes. Rapid progression to chest pain and respiratory distress leads to an almost $100 \%$ case fatality rate in humans. The incubation period in humans varies from 12 hours to 5 days, depending on the dose received, and can be longer following exposure to low doses or removal of therapeutics. Death is thought to be due to effects of the two major anthrax exotoxins, lethal toxin and edema toxin. Species differ in susceptibility, with ruminants most susceptible and carnivores generally less suscep-

DOI: 10.1097/01.LAB.0000080599.43791.01

Received May 12, 2003

Address reprint requests to: Dr. Daphne Vasconcelos, Toxicology Battelle Columbus, 505 King Avenue, Columbus, Ohio 43201. E-mail: vasconcelosd@battelle.org tible. Highly susceptible species often have minimal pathology, including splenic enlargement, widespread hemorrhages, and serous effusions. More resistant species often have lesions at the portal of entry (eg, enteritis, pharyngitis, pharyngeal lymphadenitis, or dermal necrosis and ulceration). Primates appear to have intermediate susceptibility. In addition to widespread hemorrhages, mediastinitis and hemorrhagic meningitis are prominently described in inhalation anthrax of humans. Following the recent bioterrorism events involving anthrax in the United States, there has been increased interest in the immunology of this disease and in production of new vaccines. The low availability of rhesus macaques as a model for human inhalation anthrax necessitated evaluation of more readily available nonhuman primate species as models. Cynomolgus monkeys have been used in a model of chronic industrial exposure, where lesions produced were similar to those observed in human cases of occupational exposure (Brachman et al, 1966). This paper describes the pathology associated with acute experimental inhalation anthrax in cynomolgus macaques (Macaca fascicularis).

\section{Experimental Design}

Cynomolgus monkeys were exposed to aerosolized spores of $B$. anthracis (Ames strain) in a head-only 
exposure chamber within a class III biosafety cabinet to determine the median lethal dose $\left(L D_{50}\right)$. In each stage, lethality data from the preceding stages were used to determine the doses for subsequent exposures. All monkeys that died or were killed $(n=14$, consisting of 11 female and 3 male) received a complete necropsy examination, including examination of all orifices, body cavities, and major organ systems. Mean postmortem interval was estimated to be 6 hours. Blood cultures taken from moribund monkeys (or soon after death) were used to confirm $B$. anthracis infection. Tissue samples, including all major organs and all gross lesions, were fixed in 10\% phosphatebuffered formalin and processed to hematoxylin and eosin-stained slides for microscopic examination.

\section{Results and Discussion}

\section{Clinical Observations}

Monkeys that were part of this report received between $4.56 \times 10^{4}$ and $2.94 \times 10^{6}$ colony-forming units (CFUs) of $B$. anthracis each. Monkeys generally showed few clinical signs until 1 to 2 hours prior to death, when they became progressively less responsive to external stimuli. All monkeys died within 10 days of aerosol exposure, with a tendency for those receiving higher doses to succumb more quickly than those receiving lower doses. Monkeys exposed to $>$ $2.0 \times 10^{5}$ CFUs all died within 4 days of exposure. All animals were culture-positive for $B$. anthracis.

\section{$L D_{50}$ Determination}

The probit slope was 4.21 ( $p=0.08)$, with a standard error of 2.42. The $\mathrm{LD}_{50}$ and $95 \%$ confidence intervals were $61,800(34,800$ to 110,000$)$ CFUs for an aerosol challenge of $B$. anthracis (Ames strain).

\section{Gross Pathologic Findings}

Gross findings are summarized in Tables 1 and 2. Mild splenic enlargement (1.5- to 2-fold in seven of eight affected monkeys) was the most consistently observed finding ( $57 \%$ affected). Although the degree of splenic enlargement was subjectively not remarkable, affected spleens were often noticeably soft, even in animals necropsied immediately postmortem. At least one lymph node was enlarged in eight monkeys (57\%); most commonly the bronchial or mediastinal nodes were affected (four of the eight, with at least one enlarged lymph node). Lymph node enlargement was usually mild (2- to 3-fold). Hemorrhages and edema affected a wide variety of tissues, particularly the meninges (50\%, Fig. 1), adrenal glands (29\%), and gastric mucosa (29\%). Mediastinal hemorrhage and/or edema were considered to be present when the thymus or pericardial sac was affected; this occurred in four monkeys (29\%). Fluid transudate was present within body cavities of five animals. The stomach wall of one animal was diffusely edematous in addition to having two large ecchymotic hemorrhages; in the other affected animals, small mucosal hemorrhages or focal areas of pallor and thickening (edema) of the stomach wall were present. The one animal in which intestinal hemorrhage was diagnosed had a diffusely reddened jejunum. The small intestines were diffusely fluid-filled in three monkeys (\#151, $\# 156$, and \#157). One animal (\#152) had multiple small $(5 \times 5 \times 3 \mathrm{~mm})$ dark areas of hemorrhage within the hepatic parenchyma.

\section{Histopathologic Findings}

Microscopic findings are summarized in Tables 3 and 4. Bacteria (large rods) were present intravascularly in many organs and were present extravascularly in

Table 1. Sites of Gross Hemorrhage and/or Edema in Cynomolgus Monkeys

\begin{tabular}{|c|c|c|c|c|c|c|c|c|c|c|c|c|c|c|c|}
\hline Animal identification & $101 \mathrm{M}$ & 102M & 103M & $151 \mathrm{~F}$ & $152 \mathrm{~F}$ & $153 F$ & 154F & $155 F$ & $156 \mathrm{~F}$ & $157 \mathrm{~F}$ & $158 \mathrm{~F}$ & $159 F$ & $160 F$ & $161 \mathrm{~F}$ & \\
\hline Time to death (days) & 2 & 2 & 7 & 3 & 4 & 4 & 4 & 4 & 2 & 2 & 3 & 10 & 6 & 4 & $(\%)$ \\
\hline \multicolumn{16}{|l|}{ Organ } \\
\hline Adrenal gland & - & - & - & - & + & + & - & - & - & - & - & + & + & - & $29 \%$ \\
\hline Abdomen/peritoneum & - & - & - & - & + & - & - & + & - & - & - & - & - & - & $14 \%$ \\
\hline $\begin{array}{l}\text { Body cavity, any (fluid } \\
\text { accumulation) }\end{array}$ & + & - & + & + & + & - & - & - & + & - & - & - & - & - & $36 \%$ \\
\hline Brain (Meninges) & - & - & + & - & + & + & + & + & - & - & + & + & - & - & $50 \%$ \\
\hline Heart & - & - & - & - & - & - & - & + & - & - & - & - & - & + & $14 \%$ \\
\hline Intestine & - & - & - & - & - & - & - & - & - & - & - & - & - & + & $7 \%$ \\
\hline Kidney & - & - & - & - & - & - & - & - & - & - & - & + & - & - & $7 \%$ \\
\hline Larynx & - & - & - & - & - & - & - & - & - & - & - & - & - & + & $7 \%$ \\
\hline Liver & - & - & - & - & + & - & - & - & - & - & - & - & - & - & $7 \%$ \\
\hline Lung & - & - & - & + & - & - & - & - & + & - & - & - & - & + & $21 \%$ \\
\hline Mediastinum & - & - & $++^{b}$ & - & - & - & - & $+^{a}$ & $+{ }^{b}$ & - & - & - & - & $+^{c}$ & $29 \%$ \\
\hline Pancreas & - & - & - & - & - & - & - & - & - & - & - & + & - & - & $7 \%$ \\
\hline Stomach (mucosa) & + & - & + & - & - & - & - & + & - & - & - & + & - & - & $29 \%$ \\
\hline
\end{tabular}


Table 2. Gross Enlargements of Hematopoietic Tissues in Cynomolgus Monkeys

\begin{tabular}{|c|c|c|c|c|c|c|c|c|c|c|c|c|c|c|c|}
\hline Animal identification & $101 \mathrm{M}$ & $102 \mathrm{M}$ & $103 \mathrm{M}$ & $151 F$ & $152 F$ & $153 \mathrm{~F}$ & $154 \mathrm{~F}$ & $155 \mathrm{~F}$ & $156 \mathrm{~F}$ & $157 \mathrm{~F}$ & $158 \mathrm{~F}$ & $159 \mathrm{~F}$ & $160 \mathrm{~F}$ & $161 \mathrm{~F}$ & \\
\hline Time to death (days) & 2 & 2 & 7 & 3 & 4 & 4 & 4 & 4 & 2 & 2 & 3 & 10 & 6 & 4 & $(\%)$ \\
\hline Organ & & & & & & & & & & & & & & & \\
\hline $\begin{array}{l}\text { Lymph node (axillary or } \\
\text { inguinal) }\end{array}$ & - & + & - & - & - & - & + & - & + & - & - & - & - & - & $21 \%$ \\
\hline $\begin{array}{l}\text { Lymph node (bronchial } \\
\text { or mediastinal) }\end{array}$ & - & + & - & - & + & - & + & - & - & - & - & - & - & + & $29 \%$ \\
\hline $\begin{array}{l}\text { Lymph node } \\
\text { (mesenteric) }\end{array}$ & - & - & - & - & - & - & - & + & - & - & - & - & - & - & $7 \%$ \\
\hline Lymph node (pancreatic) & - & - & - & - & + & - & - & - & - & - & - & + & - & - & $14 \%$ \\
\hline Lymph node (any) & - & + & - & - & + & - & + & + & + & - & - & + & + & + & $57 \%$ \\
\hline Spleen & + & + & - & + & + & + & - & - & + & - & - & + & - & + & $57 \%$ \\
\hline
\end{tabular}

$\mathrm{F}$, female; M, male.

more than one organ in every animal. Bacteria were recorded in the sinuses or parenchyma of bronchial lymph nodes of all animals but one. The most common microscopic finding was hemorrhage (usually small ecchymoses) in various organs but particularly in the lungs (100\% affected), bronchial and mediastinal lymph nodes (79\% and 50\% affected, respectively), meninges (57\% affected), gastrointestinal tract $(43 \%$ affected), mediastinum (36\%), heart (29\%), and adrenal glands (29\% affected). Hemorrhage in the meninges usually affected the occipital pole or was diffuse throughout the meninges. Hemorrhage in the stomach varied from ecchymotic pools to smaller mucosal extravasations. Small mucosal hemorrhages were characteristic of all hemorrhages in the intestinal tract. Congestion and edema in the lungs were present in $64 \%$ and $50 \%$ of animals, respectively. Acute suppurative inflammation was present in the lungs of four animals but was graded as minimal (barely detectable).

Lymphocyte apoptosis was characterized by presence of tingible body macrophages. Lymphocyte necrosis was characterized by presence of karyorrhectic debris without macrophages present. Both of these lesions and atrophy of lymphoid tissues were recorded together as lymphocytolysis. Lymphocytolysis was very common, affecting all animals' splenic corpuscles, splenic periarteriolar lymphoid sheaths of $93 \%$, and the germinal centers of all animals' mediastinal and bronchial lymph nodes (Fig. 7). Such lymphocytolysis was graded as at least mild in the splenic lymphoid tissue in all but one animal and in the bronchial and mediastinal lymph nodes of $57 \%$ and $36 \%$ of the monkeys, respectively; seventy-one percent of the monkeys exhibited marked lymphocytolysis in at least one lymphoid organ. Lymphocytolysis was also observed in the medullary cords and paracortices of lymph nodes, but less commonly and generally with lower severity. Minimal to mild lymphocytolysis was observed in the thymus but was considered to be consistent with spontaneous thymic involution of a juvenile cynomolgus monkey. Suppurative inflammation of lymph nodes was diagnosed when there were neutrophils within the lymph node parenchyma (ie, not only in vessels and sinusoids) or extra- capsularly. Minimal to mild suppurative inflammation was present in at least one lymph node in $50 \%$ of animals, usually in the intrathoracic lymph nodes or the mandibular lymph node. Macrophage necrosis was diagnosed in the intrathoracic lymph nodes (bronchial lymph nodes of 4 of 14 monkeys and mediastinal of 2 of 14; total 5 of 14 affected), where macrophages had pyknotic nuclei or hypereosinophilic granular cytoplasm, which often made the cytoplasmic borders indistinguishable from the surrounding granular background.

In the spleen, suppurative inflammation and fibrin thrombi were present together in 5 of 14 (36\%) monkeys, all but one of which exhibited gross splenic enlargement. In all animals but one, there were numerous polymorphonuclear leukocytes present within the liver (acute inflammation), often clustered within sinusoids. This hepatic inflammation was associated with multifocal acute hepatocellular necrosis in 5 of 14 animals (36\%).

In addition to hemorrhages, gastrointestinal tract lesions included necrosis of the tips of the villi in the jejunum or ileum in 9 of the 14 animals, suggestive of ischemic necrosis. Two of the 14 animals had inflammation associated with bacilli (consistent with vegetative $B$. anthracis) in the mucosa of the stomach. One animal had ulceration of the gastric mucosa.

Mediastinitis, denoted by the presence of neutrophils within the mediastinum in excess of what might be expected from the hemorrhage present, was observed in 4 of 14 animals (29\%, Fig. 8).

In addition to hemorrhages in the myocardium, acute suppurative inflammation was present in 4 of 14 animals (gross, Fig. 2; microscopic view, Fig. 6). Similarly, in the adrenal glands, 2 of 14 animals exhibited cortical necrosis (Fig. 9), and one of these had acute suppurative inflammation.

In the brain, necrotizing vasculitis was observed in 2 of the 14 animals and may have contributed to some of the other lesions present, including hemorrhages and leukomalacia. Leukomalacia was observed in 2 of 14 monkeys and involved the supraventricular white matter of the cerebellum (Fig. 5). Eosinophilic globules representing degenerating myelin and eosinophilic round cells with pyknotic nuclei (interpreted to be 
Table 3. Inhalation Anthrax in Cynomolgus Monkeys: Microscopic Findings in Nonlymphoid Organs

\begin{tabular}{|c|c|}
\hline & $\%$ Affected \\
\hline \multicolumn{2}{|l|}{ Adrenal glands } \\
\hline Hemorrhage & 29 \\
\hline Necrosis, cortical & 14 \\
\hline Inflammation, suppurative & 7 \\
\hline \multicolumn{2}{|l|}{ Brain } \\
\hline Bacteria, extravascular & 71 \\
\hline Necrotizing vasculitis & 14 \\
\hline Hemorrhage, meningeal & 57 \\
\hline Hemorrhage, parenchymal & 29 \\
\hline Meningitis, suppurative & 21 \\
\hline Leukomalacia & 14 \\
\hline \multicolumn{2}{|l|}{ Heart } \\
\hline Hemorrhage & 29 \\
\hline Inflammation & 29 \\
\hline \multicolumn{2}{|l|}{ Gastrointestinal tract } \\
\hline Hemorrhages, mucosal & 43 \\
\hline $\begin{array}{l}\text { Necrosis of villus tips (ileum or } \\
\text { jejunum) }\end{array}$ & 64 \\
\hline \multicolumn{2}{|l|}{ Larynx } \\
\hline Suppurative inflammation and edema & 7 \\
\hline \multicolumn{2}{|l|}{ Liver } \\
\hline Bacteria, hepatic sinusoids & 71 \\
\hline Leukocytosis/acute inflammation & 93 \\
\hline Acute necrosis & 36 \\
\hline \multicolumn{2}{|l|}{ Lung } \\
\hline Bacteria, extravascular & 79 \\
\hline Congestion & 64 \\
\hline Edema & 50 \\
\hline Hemorrhage & 100 \\
\hline Inflammation (acute, suppurative) & 29 \\
\hline \multicolumn{2}{|l|}{ Mediastinum } \\
\hline Hemorrhage & 36 \\
\hline Inflammation, suppurative & 29 \\
\hline \multicolumn{2}{|l|}{ Ovary } \\
\hline Inflammation and necrosis & 7 \\
\hline \multicolumn{2}{|l|}{ Pancreas } \\
\hline Hemorrhage & 21 \\
\hline Inflammation and necrosis & 7 \\
\hline \multicolumn{2}{|l|}{ Stomach } \\
\hline Hemorrhage, mucosal/submucosal & 29 \\
\hline Inflammation (associated with bacilli) & 14 \\
\hline Ulceration & 7 \\
\hline
\end{tabular}

either necrotic oligodendroglia or macrophages) were present within the white matter. Bacteria, some of which were encapsulated, were present within the ventricle. Suppurative meningitis was diagnosed when neutrophils were present in the meninges in numbers in excess of what seemed likely to be associated with any hemorrhage present (Fig. 4). Meningitis was present in 3 of 14 animals.

Inflammation and necrosis were also observed in the pancreas and ovary of one animal (\#155). One animal (\#161) had laryngeal inflammation with associated edema that was considered severe enough to contribute to the death of the monkey (Fig. 3).
Table 4. Inhalation Anthrax in Cynomolgus Monkeys: Microscopic Findings in Lymphoid Organs

$\%$ Affected

Lymph node, bronchial

Lymphocytolysis, germinal centers

100

Lymphocytolysis, germinal centers $>$ minimal 100

Lymphocytolysis, germinal centers $>$ mild $\quad 57$

Hemorrhage

Lymph node axillary or inguinal

Lymphocytolysis, germinal centers

93

Lymphocytolysis, germinal centers $>$ minimal $\quad 57$

Lymphocytolysis, germinal centers $>$ mild 7

Hemorrhage

Lymph node, mediastinal

Lymphocytolysis, germinal centers

100

Lymphocytolysis, germinal centers $>$ minimal $\quad 79$

Lymphocytolysis, germinal centers $>$ mild $\quad 36$

Hemorrhage

Lymph node, mesenteric

Lymphocytolysis, germinal centers

Lymphocytolysis, germinal centers $>$ minimal

Lymphocytolysis, germinal centers $>$ mild

Hemorrhage

Spleen

Lymphocytolysis, corpuscles

Lymphocytolysis, corpuscles $>$ mild

Lymphocytolysis, PALS

Lymphocytolysis, PALS > mild

Fibrin thrombi

Inflammation, suppurative

\section{Discussion}

\section{Interspecies Comparisons}

Although direct comparisons between species based on this study and previous reports are hampered by differences in study design (eg, age of animals used, strains and doses of spores, aerosol particle sizes involved, and potential pre-existing lesions), comparisons are made herein to draw attention to potential differences and similarities between the primate species. The differences described would not be statistically significant with the small sample sizes used, although they may nevertheless represent true differences in predilection for lesion development. This study suggests that rhesus and cynomolgus monkeys have a pattern of inhalation anthrax lesions largely similar to each other and to humans. Grossly, hemorrhages were common in the meninges, lung, and mediastinum of both rhesus (Fritz et al, 1995) and cynomolgus monkeys, and in at least the meninges and mediastinum of human cases (Abramova et al, 1993)). Gastrointestinal hemorrhages were observed in both rhesus (Fritz et al, 1995) and cynomolgus monkeys. Microscopically, lesions for which both nonhuman primate models appear to have an incidence similar to that in humans included acute hepatic necrosis, acute pulmonary inflammation, and lymphocytolysis within the lymph nodes and spleen (incidence approached $100 \%$ in all three species) (Fritz et 


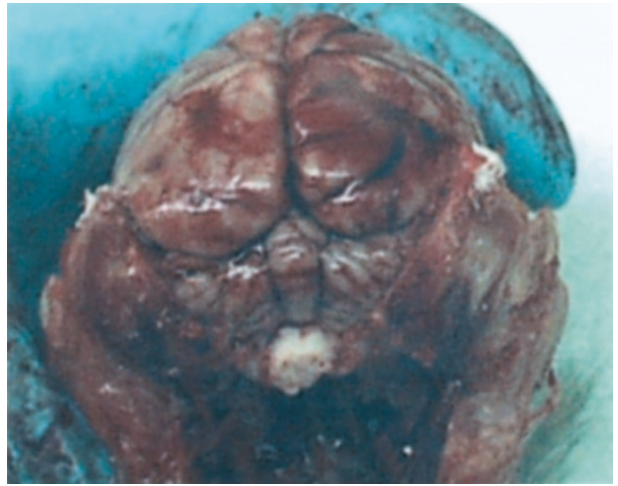

Figure 1.

Brain from monkey \#152, exhibiting moderate meningeal hemorrhage characteristic of septicemia following aerosol challenge with spores of Bacillus anthracis.

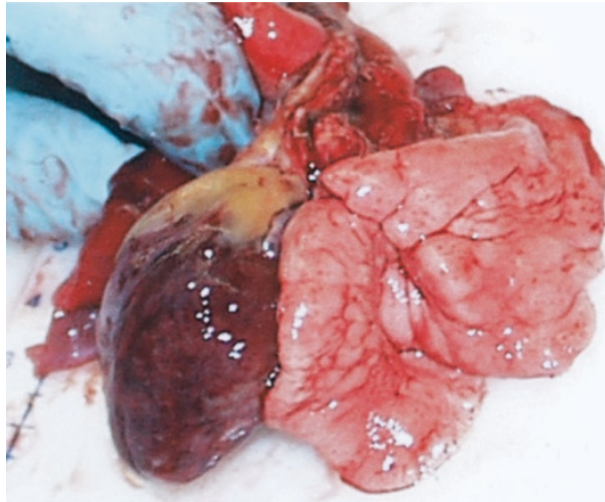

Figure 2.

Heart and lungs from monkey \#155. There is extensive epicardial hemorrhage and edema within the anterior mediastinum and thymus.

al, 1995; Grinberg et al, 2001). Among the human cases at Sverdlovsk, $16 \%$ had overt centrilobular necrosis, whereas $23 \%$ had morphologic evidence of increased apoptosis in the liver (Grinberg et al, 2001). Although overt coagulation necrosis was by far the more common of these two manifestations of hepatic damage among the cynomolgus monkeys, the proportion affected (36\%) is comparable to that in humans. Multifocal necrotizing vasculitis was observed in all three species.

Of the gross lesions, the greatest differences between this study and that of Fritz et al (Fritz et al, 1995) were in the incidence of lymph node enlargement: Fritz et al recorded enlarged lymph nodes in $85 \%$ of rhesus monkeys, whereas we recorded enlarged nodes in only $57 \%$. Because there is considerable normal size variation in the lymph nodes of young monkeys, many of the lymph nodes in our study were not considered to be outside the normal size limits. Microscopically, the incidence of hemorrhage and edema in the cynomolgus monkeys ( $\geq 79 \%$ ) was similar to that in the rhesus monkeys $(77 \%)$, and both were less than is reported in humans (94\%) (Fritz et al, 1995; Grinberg et al, 2001).

Splenomegaly was recorded in $57 \%$ of the cynomolgus monkeys, although in most cases it was mild

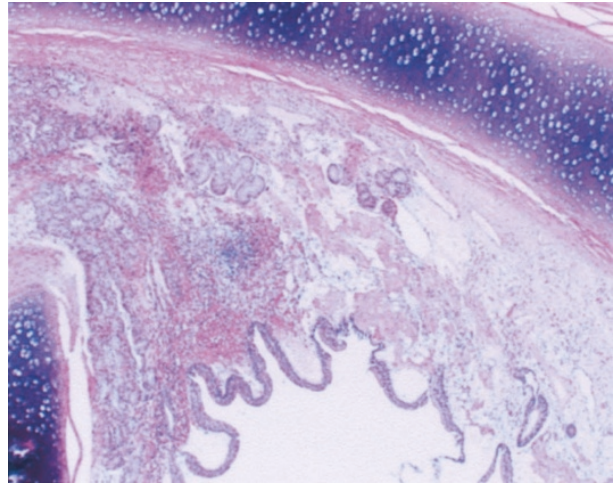

Figure 3.

Larynx from monkey \#161 showing edema, hemorrhage, and lymphocytolysis in the submucosa. The cricoid cartilage is at top and the arytenoid cartilage at lower left. Hematoxylin and eosin staining, $\times 40$ magnification.

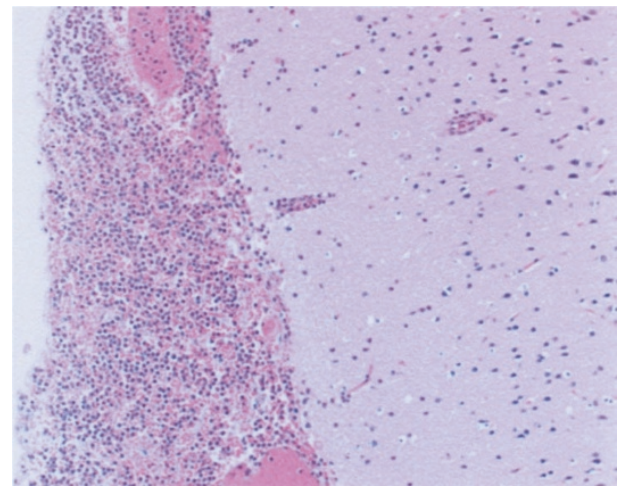

Figure 4.

Cerebrum from monkey \#154 has meningeal hemorrhage and infiltration of meninges with numerous neutrophils. Hematoxylin and eosin staining, $\times 100$ magnification.

(1.5- to 2-fold in seven of eight affected animals). This is also comparable to Gleiser et al's report (Gleiser et al, 1963) that splenomegaly was frequently seen, although it is apparently not a common finding in humans (Abramova et al, 1993; Grinberg et al, 2001). Only one of the affected animals in this study was killed; the splenomegaly in that case may have been due to barbiturate administration. Cardiac lesions were seldom reported in rhesus monkeys (Fritz et al, 1995; Gleiser et al, 1963) but were present in two of the cynomolgus monkeys.

Microscopically, meningitis appeared to be approximately twice as common among rhesus monkeys as among humans, and the incidence among cynomolgus monkeys was approximately half of that reported in humans (Fritz et al, 1995; Grinberg et al, 2001). However, meningitis was only recorded in the cynomolgus monkeys when the number of leukocytes, particularly neutrophils, exceeded the number expected from the hemorrhage that was commonly present (ie, to distinguish between hemorrhage alone and meningitis). This appears similar to the criteria used in diagnosing meningitis in the rhesus monkeys (Fritz et al, 1995). However, Grinberg et al (2001) recorded the presence of various types of leukocytes 


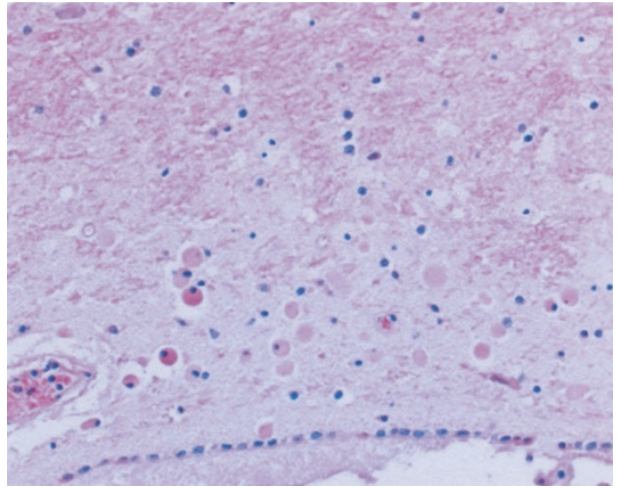

Figure 5.

Cerebellar white matter and fourth ventricle from monkey \#156. The white matter is vacuolated and contains cells with pyknotic nuclei and cytoplasmic eosinophilia. Hematoxylin and eosin staining, $\times 200$ magnification.

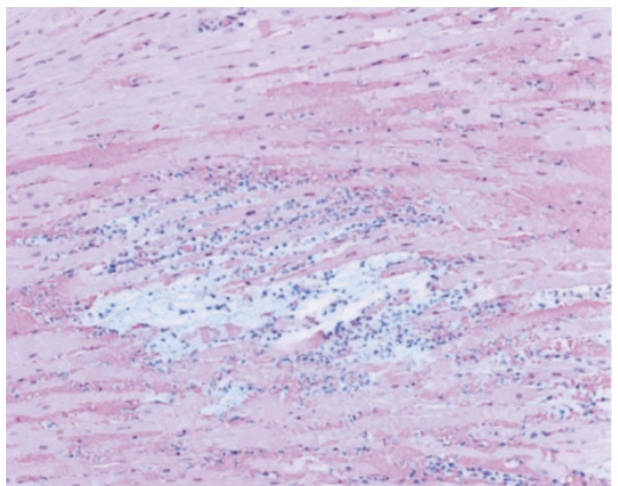

Figure 6.

Heart (papillary muscle) from monkey \#155 that had multiple areas of necrosis and lymphohistiocytic inflammation, with numerous unidentified cocci and fewer bacilli characteristic of Bacillus anthracis. Hematoxylin and eosin staining, $\times 100$ magnification.

rather than diagnosing meningitis per se, leaving the actual incidence of meningitis open to interpretation. Because the average severity reported was 0.5 (ie, "present but barely detectable"), this probably overestimates the number of cases that would be diagnosed as meningitis in either nonhuman primate study. As a result, the actual percentage of human cases with meningitis by our criteria may be closer to the incidence in cynomolgus monkeys than in rhesus.

Mediastinal hemorrhage and inflammation were less frequently reported in the nonhuman primates than in humans from the Sverdlovsk epidemic (Grinberg et al, 2001). As in the present report, Fritz recorded mediastinal changes in $30 \%$. However, if capsulitis and hemorrhage of the bronchial and mediastinal lymph nodes are included as mediastinal inflammation and hemorrhage, the incidence of mediastinal lesions becomes similar in all three species. Gleiser et al (1963) noted in rhesus monkeys, as also noted in the present report in cynomolgus monkeys, that mediastinal hemorrhage, edema, and inflammation were minimal and almost always associated with more extensive lesions in the intrathoracic lymph nodes. He also inferred that the mediastinal changes were most likely secondary to the lymph node lesions. Thus, the apparent discrep-

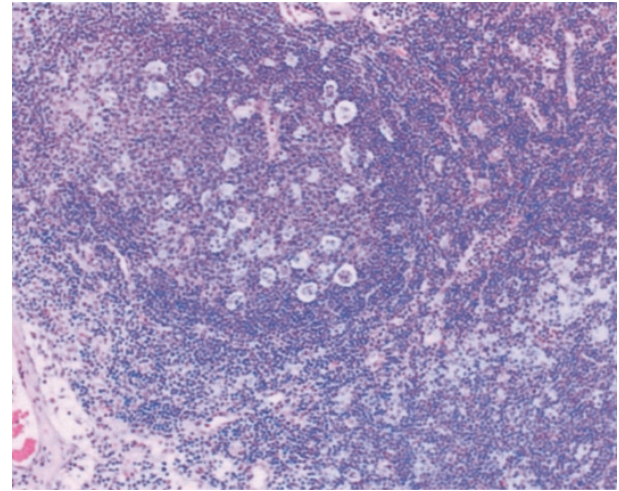

Figure 7.

A mediastinal lymph node from monkey \#161 demonstrates lymphocytolysis of germinal centers and relative sparing of the paracortex. The capsule is at left. Hematoxylin and eosin staining, $\times 100$ magnification.

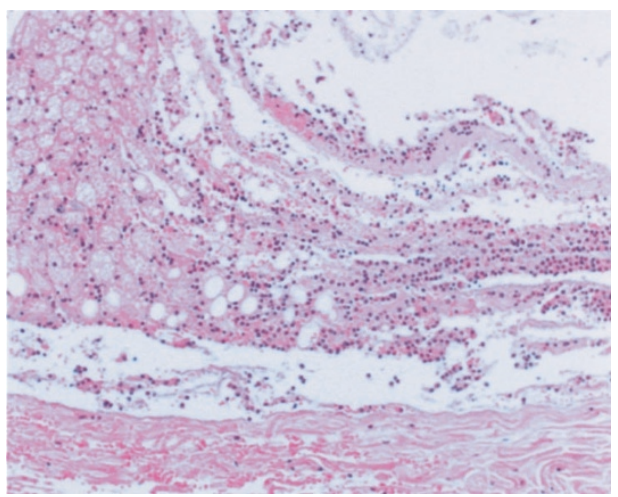

Figure 8.

Mediastinum from monkey \#102, infiltrated by neutrophils and erythrocytes. Mediastinal fat is at left. Hematoxylin and eosin staining, $\times 100$ magnification.

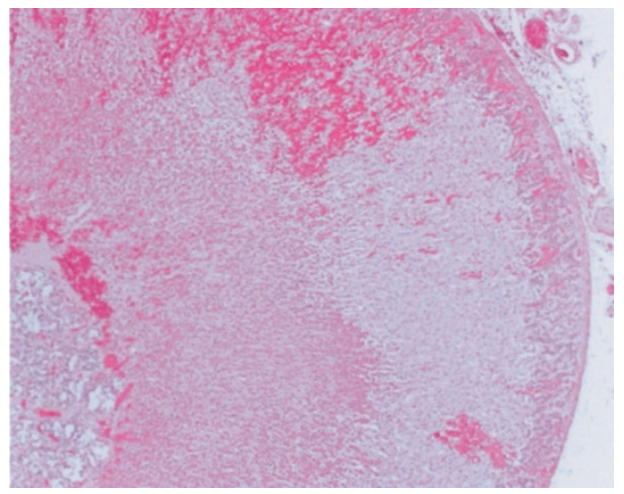

Figure 9.

Adrenal gland from monkey \#159. Capsule is at right and medulla at left. There is a band of necrosis involving the zona fascicularis and zona reticulata extending from the corticomedullary junction. The outer zona fascicularis and the zona glomerulosa are spared. Sinusoidal dilation and hemorrhage are visible at top, bottom right, and at the corticomedullary junction. Hematoxylin and eosin staining, $\times 40$ magnification.

ancy between incidences of mediastinal lesions between species may be a difference in terminology and reporting of lesion sites between authors, rather than a true difference in lesion incidence. In addition we observed that affected bronchial and mediastinal 
lymph nodes often became so hemorrhagic that their identification was based on the presence of a few residual lymphocytes and a capsule remnant. It is possible that lymph node lesions of greater severity might cause disruption of the capsule and total loss of the normal architecture, and hence be recorded as mediastinal in origin.

Lesions for which cynomolgus monkeys appear to have incidences more similar to humans than do rhesus monkeys (ie, lesions for which cynomolgus monkeys more closely simulate the human disease) include the following: hemorrhages of the adrenal glands, hepatic inflammation (each 4-fold higher than reported for rhesus, and comparable to that reported in humans), and pulmonary edema and hemorrhage (30\% of rhesus, $100 \%$ of cynomolgus, and $66 \%$ to $86 \%$ of humans) (Fritz et al, 1995; Grinberg et al, 2001).

Cardiac inflammation was more common in cynomolgus monkeys than in rhesus and was not reported in the human cases. This may represent a real difference in organ susceptibility between species. One of the animals with myocarditis (\#155) had abundant cocci associated with the inflammation, although anthrax bacilli were also present, and only $B$. anthracis was cultured.

Some of the lesions present appeared to be a more common finding in monkeys with a longer disease progression, ie, in those that survive longer postchallenge. Adrenal edema and hemorrhage began to appear by 4 days postchallenge, and their incidence increased among the few animals surviving longer. Adrenal inflammation and necrosis were only present in those animals living more than 4 days. Similarly, adrenal lesions were present in $62 \%$ of the monkeys that died following chronic exposure in the study by Brachman et al (1996). Meningeal hemorrhage was more common in those animals surviving at least 4 days, and cerebral vasculitis was only observed among animals surviving 4 days. The incidence of meningeal hemorrhage in cynomolgus monkeys dying of anthrax following chronic exposure was reported as $43 \%$, quite similar to the incidence in this study (Brachman et al, 1966). Hepatic necrosis also occurred with increasing incidence commencing on Day 4 postchallenge. The incidence reported for hepatic necrosis in cynomolgus monkeys dying following chronic exposure was almost identical (33\%) (Brachman et al, 1966). Surprisingly, however, these are all lesions for which the incidence reported herein was higher than that previously reported for rhesus monkeys, despite the fact that the mean survival time in this study was only 4.1 days, compared with 5.5 days in the rhesus report (Fritz et al, 1995), suggesting true differences in incidence between species.

Unusual lesions of anthrax reported herein include cerebellar leukomalacia, which was deemed to be secondary to vasculitis even though vasculitis was not diagnosed in the cerebral vessels of all animals with leukomalacia. Adrenal necrosis was described previously by Gleiser et al (1963) but appears uncommon in all primates. Gastric ulceration has been previously described in an anthrax-infected rhesus monkey, although the associated massive bacterial proliferation seen in the present cynomolgus case was not described; however, infarcts associated with bacterial proliferation have been previously described in the large intestine (Fritz et al, 1995; Gleiser et al, 1963). Macrophage necrosis, though a described effect of anthrax toxins in vitro (Hanna et al, 1993), does not appear to have been morphologically described previously (Fritz et al, 1995; Gleiser et al, 1963; Grinberg et al, 2001). Renal tubular casts were observed in one animal in this study compared with the presence of cellular casts in 4 of 41 affected people; however, the casts observed in the animal in this study were protein casts rather than erythrocytic casts. Protein casts are fairly common spontaneous findings among laboratory monkeys, and hence may not be related to infection with $B$. anthracis. Myocarditis, and ovarian and pancreatic necrosis and inflammation, were observed in an animal that appeared to have a mixed septicemia (\#155). Although the slightly unusual spectrum of changes may have been due to the presence of other bacteria, it is worth noting that mixed infections are a potential complication of anthrax infection.

All animals except the one described above (\#155) appeared to die directly from anthrax septicemia/ toxemia. Monkey \#155 had abundant cocci in the heart and other organs, in addition to modest numbers of large rods interpreted to be $B$. anthracis. As it is exceedingly rare for a laboratory monkey to spontaneously develop and die from septicemia, it seems likely that this monkey developed a mixed infection subsequent to development of anthrax septicemia. Previous reports demonstrate that a high proportion of irradiated mice develop mixed infections with enteric organisms when challenged with $B$. anthracis (Brook et al, 2001), suggesting that $B$. anthracis enhances translocation of organisms from the gastrointestinal tract into the bloodstream, possibly by causing vasculitis.

Not all animals had gross lesions suggestive of anthrax: one had only meningeal hemorrhage (\#158) and one had fluid-filled intestines and no other gross lesions (\#157). This suggests that without microscopic examination, such inhalation anthrax cases could be missed.

\section{Lymph Node Lesions and Pathogenesis}

A comparison of severities of lymphocytolysis among germinal centers of various sites (Table 4) reveals two major patterns. Intrathoracic lymph nodes (bronchial and mediastinal) and splenic lymphoid tissues were frequently affected by moderate to marked lymphocytolysis, whereas other lymphoid tissues were commonly only minimally to mildly affected. Minimal to mild lymphocytolysis is commonly seen in lymphoid tissues due to corticosteroid release secondary to the stress of disease, and hence may well be an indirect result of infection. Thus, the intrathoracic lymph nodes and spleen may be considered to be targets of inha- 
lation anthrax, whereas other lymphoid tissues are probably not.

There have been conflicting reports regarding whether $\mathrm{T}$ or $\mathrm{B}$ cell-rich areas are affected more by anthrax: Fritz et al recorded more damage to B cellrich zones, whereas Grinberg et al stated that $T$ cell-rich zones were somewhat more affected (Fritz et al, 1995; Grinberg et al, 2001). With the exception of the splenic periarteriolar lymphoid sheaths in the present study, B cell-rich zones appeared more severely affected. The absence of thymic lesions supported this finding. The animals in the present study were young and had thymic tissue that was essentially normal. That is, the minimal to mild apoptosis observed is commonly seen in pubescent monkeys either as the thymus begins to involute or due to corticosteroid release from a variety of causes. Such sparing of the thymus was also noted by Gleiser et al (1963), who used rhesus monkeys of 2 to $4 \mathrm{~kg}$, which were likely to be even younger than those used in our study. Brachman et al (1966) used monkeys of approximately $3 \mathrm{lbs}$, likely to be of similar age to those in the present study, yet he made no comment regarding thymic lesions, although mediastinal edema was considered one of the most consistent lesions present. Fritz et al (1995) did not mention thymic lesions; however, the animals in Fritz et al's study were larger than those in the present study $(6.0$ to $16.8 \mathrm{~kg}$ compared with 2.4 to $7.4 \mathrm{~kg}$ in the present study). Even though rhesus monkeys are a larger species than cynomolgus monkeys, these weights suggest that a greater proportion of the rhesus monkeys were adult and would have already had complete thymic involution. Thymic involvement in human cases does not appear to have been described. However, reports of human cases have largely described adults in whom thymic tissue would be scant (Abramova et al, 1993; Grinberg et al, 2001). The apparent predilection for B cell-rich areas and sparing of the thymus suggest that lymphocyte populations may have heterogeneous susceptibilities to anthrax and the mediators of anthrax-induced lesions (toxins and cytokines). This may provide an area for future research regarding the pathogenesis of inhalation anthrax.

\section{Materials and Methods}

\section{Animals}

Cynomolgus monkeys were exposed to spores of $B$. anthracis (Ames strain) in a head-only exposure chamber as part of a study to determine the $L D_{50}$ of $B$. anthracis by inhalation route. In each stage, lethality data from the preceding stages were used to determine the doses for subsequent exposures. All monkeys were juvenile (1.7 to $3.8 \mathrm{~kg}$ ) Macaca fascicularis obtained from Battelle primate holding facility. All monkeys were negative by enzyme-linked immmunosorbent assay for herpes B virus, had negative tuberculin tests, and were seronegative for simian immunodeficiency virus, simian T-lymphotropic virus-1, and simian retrovirus. Monkeys were pair- housed in a facility fully accredited by the International Association for the Assessment and Accreditation of Laboratory Animal Care and registered with the United States Department of Agriculture as a Research Facility (Number 31-R-021). All procedures and care were carried out by trained personnel in accordance with guidelines set forth in the "Guide for the Care and Use of Laboratory Animals" (National Research Council, National Academy Press, 1996) and in the regulations and standards of the Agricultural Research Service, United States Department of Agriculture, pursuant to the Animal Welfare Act of August 24, 1966, as amended. Monkeys were pair-housed, fed commercially available monkey chow (PMI, Inc., Certified Monkey Chow), and were provided with enrichment opportunities to promote and enhance their psychological well being. Each monkey was examined by a veterinarian prior to inclusion in the study and again prior to aerosol exposure and found to be free of malformations or clinical signs of disease.

\section{Exposures}

Aerosol exposure was performed in an exposure box located within a class III biological safety cabinet, under Telazol anesthesia (3 to $6 \mathrm{mg} / \mathrm{kg} \mathrm{im}$ ), and was followed by 3 to 5 minutes of fresh air. Ames strain of $B$. anthracis was used. The monkeys' heads were decontaminated prior to removal of the animals from the cabinet. Aqueous suspensions of $B$. anthracis were aerosolized by a three-jet collision nebulizer. Samples of the aerosol stream were assayed to determine CFUs per milliliter. The mass median aerodynamic diameter of the generated particles for all exposures was between 1 and $2 \mu \mathrm{m}$ as determined by an API Aerosizer (Amherst Process Instruments, Inc., Hadley, Massachusetts) or Aerodynamic Particle Sizer (TSI, Inc., St. Paul, Minnesota). Respiratory rates, tidal volumes, and minute ventilation were determined by plethysmography for each monkey before and during aerosol challenge. All exhausts were filtered through HEPA filters before exhausting into the class III biosafety cabinet and then double HEPA-filtered before leaving the building. Following challenge, all monkeys were observed at least twice daily (morning and evening) for signs of illness or death, and were killed according to predetermined criteria approved by the Battelle Institutional Animal Care and Use Committee. Euthanasia was by injection with an agent containing pentobarbital following anesthesia with Telazol (3 to 6 $\mathrm{mg} / \mathrm{kg} \mathrm{im}$ ). Blood cultures obtained immediately prior to euthanasia or prior to necropsy (for animals found dead) were used to confirm $B$. anthracis infection.

\section{$L D_{50}$ Determination}

The primate model of inhalation anthrax is based on the calculated number of inhaled spores. A stage-wise approach was used to determine the $\mathrm{LD}_{50}$ to within a target $0.5-\log$ dilution. Preliminary lethality results from all preceding stages were used to select doses for subsequent stages. A probit dose-response model 
was fitted to the lethality data combined over both sexes and over the experiment dates, as a function of total inhaled dose (CFU). The resulting probit curve was used to estimate the $L D_{50}$. The probit model was fitted using the SAS (version 8.2) PROBIT procedure.

\section{Gross Pathology}

All monkeys that died or were killed in moribund condition ( $n=14,11$ female and 3 male) received a complete necropsy examination by an ACVP board-certified veterinary pathologist. Necropsies included examination of all orifices, body cavities, and major organ systems. Mean postmortem interval was estimated to be 6 hours, and ranged from 0.5 hours to 14.5 hours.

\section{Histopathology}

Samples of the following tissues were fixed in $10 \%$ phosphate-buffered formalin, processed to 5 - $\mu$-thick sections, and stained with hematoxylin and eosin: adrenal glands, brain, epididymides, esophagus, eyes, gallbladder, heart, intestines (duodenum, jejunum, ileum, cecum, colon, and rectum), kidneys, liver, lungs, lymph nodes (axillary, bronchial, inguinal, mandibular, mediastinal, and mesenteric), mammary gland, muscle, nerve (sciatic), ovaries, pancreas, pituitary, prostate, salivary glands, seminal vesicles, spleen, sternum with marrow, stomach, testes, thymus, thyroids with parathyroids, trachea, urinary bladder, uterus with cervix, and all gross lesions. Lesions were graded semiquantitatively as follows: a severity score of 1 represented a barely detectable alteration (minimal), a severity score of 2 represented a mild alteration likely to have functional or subclinical significance, a severity score of 3 represented a moderate change likely to have clinical significance, and a severity score of 4 represented a marked alteration approaching the maximal extent possible in that organ. Diagnoses were recorded in the Xybion PATH/TOX data management system for production of tables and storage of data. All data and slides were peer-reviewed by a second ACVP board-certified pathologist.

\section{Acknowledgements}

We thank Kandy Audet, William Hart, Hubina Hirst, Tracy Hoff, Robert Jarvis II, Pamela Kinney, and Melanie (Autumn) Smiley for necropsy support and animal care; Connie Essman-Wood for histology coordination; Craig Ault, Jay Bailey, Marijo Cistone, Pauletta Cochrane, Dave Cook, Padmini Fernando, Zachary King, and Seth Walker for histologic preparations; and Michael Ryan for peer review. Supported by the Defense Advanced Research Projects Agency's Defense Sciences Office (DARPA/DSO) anthrax therapeutics acceleration program. Approved for public release.

\section{References}

Abramova FA, Grinberg LM, Yampolskaya OV, and Walker DH (1993). Pathology of inhalational anthrax in 42 cases from the Sverdlovsk outbreak of 1979. Proc Natl Acad Sci USA 90:2291-2294.

Brachman PS, Kaufman AF, and Dalldorf FG (1966). Industrial inhalation anthrax. Bacteriol Rev 30:646-659.

Brook I, Elliott TB, Harding RA, Bouhaouala SS, Peacock SJ, Ledney GD, and Knudson GB (2001). Susceptibility of irradiated mice to Bacillus anthracis Sterne by the intratracheal route of infection. J Med Microbiol 50:702-711.

Fritz DL, Jaax NK, Lawrence WB, Davis KJ, Pitt MLM, Ezzell JW, and Friedlander AM (1995). Pathology of experimental inhalation anthrax in the Rhesus monkey. Lab Invest 73:691702.

Gleiser CA, Berdjis CC, Hartman HA, and Gochenour WS (1963). Pathology of experimental respiratory anthrax in Macaca mulatta. Br J of Experimental Pathology 44:416426.

Grinberg LM, Abramova FA, Yampolskaya OV, Walker DH, and Smith JH (2001). Quantitative pathology of inhalational anthrax I: Quantitative microscopic findings. Mod Pathol 14:482-495.

Hanna PC, Acosta D, and Collier RJ (1993). On the role of macrophages in anthrax. Proc Natl Acad Sci USA 90:1019810201. 